\title{
Negative Ion Production and Beam Extraction Processes in a Large lon Source ${ }^{a)}$
}

\author{
K. Tsumori ${ }^{1,2}$, K. Ikeda ${ }^{1}$, H. Nakano ${ }^{1,2}$, M. Kisaki ${ }^{1}$, S. Geng ${ }^{2}$, M. Wada $^{3}$, \\ K. Sasaki ${ }^{4}$, S. Nishiyama ${ }^{4}$, G. Serianni ${ }^{5}$, C. Wimmer ${ }^{6}$, P. Agosttineti ${ }^{5}$, \\ E. Sartori ${ }^{5}$, M. Brombin ${ }^{5}$, K. Nagaoka ${ }^{1,2}$, M. Osakabe ${ }^{1,2}$, Y. Takeiri ${ }^{1,2}$, \\ O. Kaneko ${ }^{1,2}$, and NIFS NBI group ${ }^{1}$ \\ ${ }^{I}$ National Institute for Fusion Science, 322-6 Oroshi Toki Gifu 509-5292, Japan \\ ${ }^{2}$ The Graduate University for Advanced Studies, Shonan Village, Hayama, Kanagawa 240-0193, Japan \\ ${ }^{3}$ Graduate School of Science and Engineering, Doshisha University, Kyotanabe, Kyoto 610-0321, Japan \\ ${ }^{4}$ Division of Quantum Science and Engineering, Hokkaido University, Sapporo 060-8628, Japan \\ ${ }^{5}$ Plasma Engineering Group, Consorzio RFX - Corso Stati Uniti, 4, 35127 Padova, Italy \\ ${ }^{6}$ Max-Planck-Institut für Plasmaphysik, Bereich ITER-Technologie \& -Diagnostik / N-NBI Boltzmannstr. \\ 2, 85748 Garching, Germany \\ Corresponding Author: Katsuyoshi Tsumori, e-mail address: tsumori@nifs.ac.jp
}

(Presented XXXXX; received XXXXX; accepted XXXXX; published online XXXXX)

(Dates appearing here are provided by the Editorial Office)

\begin{abstract}
Recent research results on negative-ion-rich plasmas in a large negative ion source have been reviewed. Spatial density and flow distributions of negative hydrogen $\left(\mathrm{H}^{-}\right)$ions and positive hydrogen ions together with those of electrons are investigated with a 4-pin probe, and a photodetachment (PD) signal of a Langmuir probe. The PD signal is converted to local $\mathrm{H}^{-}$density from signal calibration to a scanning cavity ring down $\mathrm{PD}$ measurement. Introduction of $\mathrm{Cs}$ changes the slope of plasma potential distribution depending upon the plasma grid bias. Higher electron density included in $\mathrm{H}_{2}$ plasmas shields bias potential compactly and behaves like free electrons in metals. On the other hand, the bias and extraction electric fields penetrate in Cs-seeded electronegative plasmas even in the similar plasma density. Electrons are possibly pulled with the penetrated field from driver region along linkage field of filter and electron deflection magnets. Electrons and ions show the clearly different responses to extraction field near the boundary of the magnetic domains induced with filter and electron deflection magnets.
\end{abstract}

\section{INTRODUCTION}

High power beam injections of several MW are required for plasma heating and current drive in magnetically confinement fusion machines near future ${ }^{1-3}$. Injection stability is one of the important issues for such powerful injections. Experiments using deuterium plasmas are scheduled in $\mathrm{NIFS}^{4}$ and neutral beam based on deuterium negative ion $\left(\mathrm{D}^{-}\right)$beams will be injected. According to Child-Langmuir's low, current density of $\mathrm{D}^{-}$ beam at the same induced electric field can be $1 / \sqrt{2}$ times lower than negative hydrogen ion $\left(\mathrm{H}^{-}\right)$beam because of the isotope effect. Plasma performance of Large Helical Device (LHD) is strongly affected by the NBI power, and the same level of power as $\mathrm{H}^{-}$beam is required for $\mathrm{D}^{-}$beam.

In order to improve the efficiency of $\mathrm{H}^{-}$yield, we have continuously investigated particle dynamics of the plasmas in beam extraction region ${ }^{5-9}$. It was found that $\mathrm{H}^{-}$rich plasmas are generated in a wide region of $\mathrm{Cs}$ seeded extraction plasma, but their density ratios of $\mathrm{H}^{-}$to electron decrease due to electron diffusion from driver region when the beam extraction field is induced. To deepen our understanding in physics of negative ion rich plasmas, temperature measurements of neutral hydrogen atoms $\left(\mathrm{H}^{\circ}\right)$ and those of $\mathrm{H}^{-}$as well as investigations on flows of charged particles have been started recently. New results on formation of beamlets and their interaction have been also obtained. In this paper, we report the recent collaboration research activities on $\mathrm{H}^{-}$production and beam extraction using NIFS-R\&D negative ion source.

\section{NIFS-R\&D SOURCE AND ITS DIAGNOSTIC SYSTEM}

Details of NIFS-R\&D negative ion source (NIFSRNIS), or the source previously called $1 / 3$ scaled negative ion source, are described elsewhere ${ }^{5,6}$. The source is a magnetic multi-cusp plasma container with a pair of filter magnets to form magnetic field strength of $6.3 \mathrm{mT}$ at the center of plasma grid (PG). The inner size of the source is $350 \mathrm{~mm}$ in width $\times 230 \mathrm{~mm}$ in depth $\times 700 \mathrm{~mm}$ of height, which is a half the height of LHD negative ion source.

${ }^{a}$ Invited paper published as part of the Proceedings of the 16th International

Conference on Ion Source, New York, USA, August, 2015.

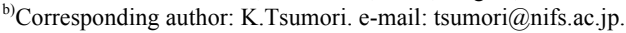




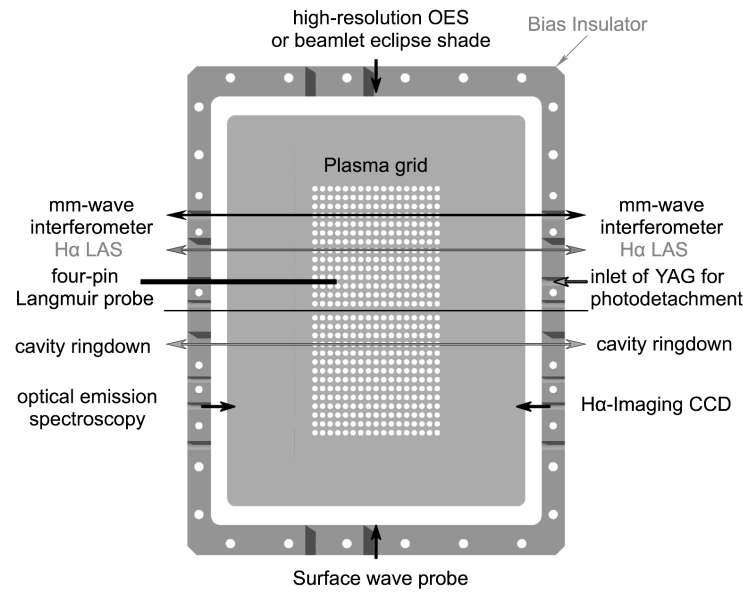

FIG. 1. Arrangement of diagnostic modules installed at bias insulator surrounding the PG viewing from back plate of R\&D negative ion source.

By replacing the original bias insulator to a thicker one with several ports, diagnostic modules are attached to monitor the plasmas in beam extraction region between PG and the filter field. The arrangement of the diagnostic modules is shown in Fig. 1 as a view from the back plate of the source. Among these modules, cavity ringdown (CRD), four-pin directional Langmuir probe and surface wave probe are movable and can obtain the spatial distributions of target parameters. Data obtained by most of the modules are acquired with $\mathrm{A} / \mathrm{D}$ convertor connected a computer at high voltage stage of the ion source and raw data are sent via an optical fiber cable to ground station computers. This allows the data acquisition even during beam acceleration.

\section{EXPERIMENTAL RESULTS}

\section{A. Plasma potential distribution}

The plasma potential profile determined by PG bias changes energy of charged particles, and forms local electric field that affects $E \times B B$ drift. These quantities are important to estimate the motions of electrons and ions from a single particle viewpoint. Distributions of plasma potential measured in pure $\mathrm{H}_{2}$ discharge by a Langmuir probe are shown in Fig. 2(a) for different PG bias voltages applied with respect to the chamber wall. In the figure, the origin of the horizontal axis corresponds to PG surface
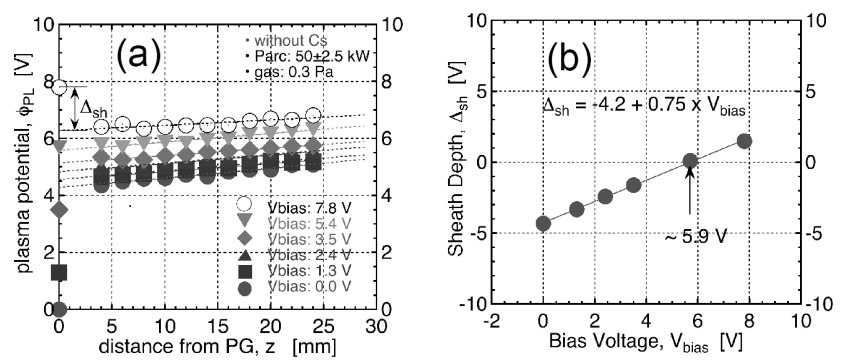

FIG. 2. (a) Plasma potential distribution in $\mathrm{H}_{2}$ plasma with different $\mathrm{PG}$ bias voltages, and (b) dependence of sheath gap, $\Delta_{\text {sh }}$, upon PG bias. facing the plasma. The slope of the potential distribution is nearly constant against the distance from the PG, $z$, and the slope does not change against the $\mathrm{PG}$ bias. The potential difference between the PG bias and the extrapolation of the slope up to $z$ origin is designated as a sheath gap $\left(\Delta_{\mathrm{sh}}\right)$, which also exhibits a simple linear correlation to the PG bias voltage as shown in Fig. 2(b). The line intersects at $5.9 \mathrm{~V}$ while the slope is 0.75 instead of 1 suggesting that the electric field in the extraction region is affected by the PG bias.
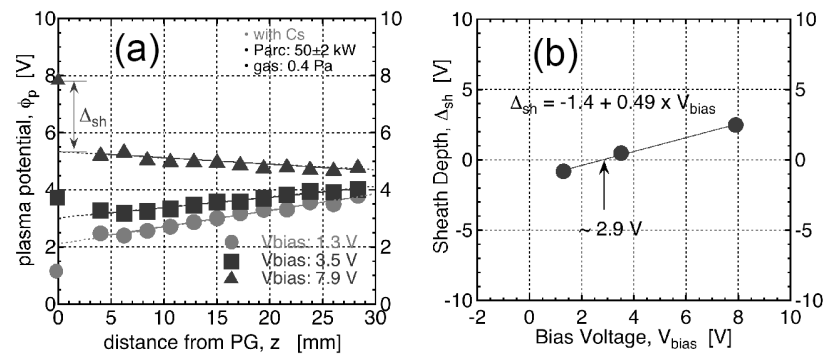

FIG. 3. (a) Plasma potential distributions in Cs seeded plasmas for different PG bias, and (b) dependence of sheath gap, $\Delta_{\text {sh }}$, upon PG bias.

In the case of $\mathrm{H}^{-}$-rich plasma, the slope responds to bias voltage more sensitively than the case of $\mathrm{H}_{2}$ plasma case as indicated in Fig. 3(a). Although the sheath gap, $\Delta_{\mathrm{sh}}$, appears linear to bias voltage as well as the case of $\mathrm{H}_{2}$ discharge, the proportional constant is as small as 0.49 as shown in Fig. 3(b). The sheath gap voltage is smaller, and the plasma behaves electrically resistive to build up electric field in the plasma with the certain distance from PG. Sheath formation and response to external electric field depends on the mobility of the dominant charge carrier. The difference of potential distributions in Figs. 2(a) and 3(a) is possibly explained by the dominant negative charge species, electrons or ions, in the plasma.

\section{B. Probe saturation current}

By applying a beam extraction voltage, electron component increases the negative saturation current in $\mathrm{H}^{-}$ rich plasma. Comparison of spatial distribution of the current before and during the beam extraction possibly enables one to obtain a clue how electrons flow in the plasma. Figure 4(a) shows distribution of saturation currents before and during the beam extraction in Cs seeded plasma with the probe scanning direction set parallel to the PG plane and perpendicular to the aligned rows of the electron deflection magnets (EDM) imbedded in extraction grid (EG). Input arc power, operational $\mathrm{H}_{2}$ pressure and bias voltage are $50 \mathrm{~kW}, 0.3 \mathrm{~Pa}$ and $-4 \mathrm{~V}$, respectively. The distance of probe tip is $4 \mathrm{~mm}$ from PG surface. Negative and positive saturation currents become larger as moving the probe tip from aperture region to the cusp-line of $\mathrm{EDM}^{10}$. The negative saturation current increases during extraction. Ratios of saturation currents during extraction to the current before extraction are 


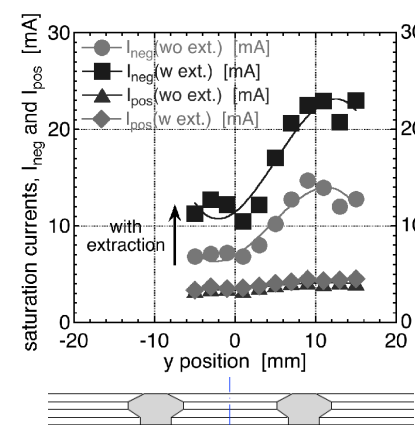

cross section of $\mathrm{PG}$

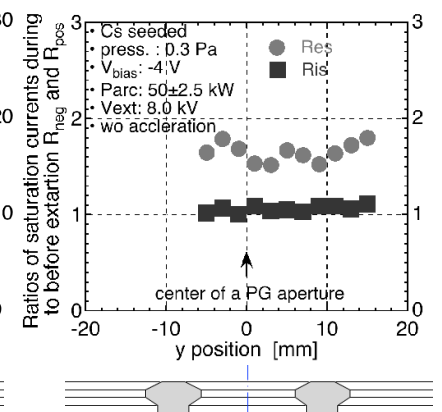

cross section of $\mathrm{PG}$
FIG. 4. (a) Distribution of saturation currents of the probe scanned parallel to PG before and during extraction, and (b) ratios of the saturation currents during extraction to that before extraction.

shown in Fig. 4(b). It is noticeable that the positive saturation current is unity and negative saturation current (electron current) increases almost equally from the part above an aperture to the part above blocking electrode.

\section{Temperature of hydrogen atoms}

High-resolution optical emission spectroscopy ${ }^{11}$ (HROES) and $\mathrm{H} \alpha$ laser absorption spectroscopy ${ }^{12}$ (H $\alpha$ LAS) are applied to measure the temperature of $\mathrm{H}^{0}$ in the extraction region. Wave precision and resolution of spectrometer applied for HR-OES are $1 \mathrm{pm}$ and $10 \mathrm{pm}$, respectively. Balmer- $\alpha$ light emission from the extraction region is collimated and sent via optical fiber to the spectrometer. The inlet of the optical fiber is set at the top of the bias insulator as shown in Fig. 1.

Changes of Balmer- $\alpha$ spectrum due to the bias voltage increasing from 0 to $+10 \mathrm{~V}$ is shown in Fig. 5. The Balmer- $\alpha$ line width reduces continuously with the increasing bias voltage, and shift in of the blue-wing causing this narrower spectrum broadening. Positive ions are bent with filter field from driver region to the HR-OES line of sight. Assuming the contributions of hydrogenous positive ions $\left(\mathrm{H}_{\mathrm{m}}{ }^{+}, \mathrm{m}=1,2\right.$ and 3$)$, the result is explainable to bias dependence as well as the blue shift. In the situation with low electron temperature and $\mathrm{H}^{-}$-rich plasma, dominate processes emitting Balmer- $\alpha$ and related to positive ions are follows ${ }^{13,14}$ :

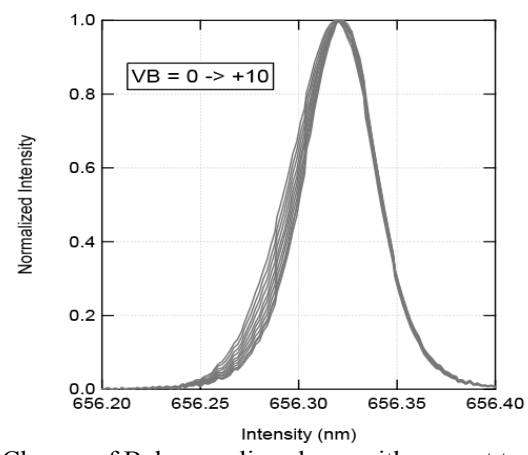

FIG. 5. Change of Balmer- $\alpha$ line shape with respect to bias voltage.

$$
\begin{aligned}
& H_{2}^{+}+e \rightarrow H(n=3)+H(n=1) \\
& H^{+}+H^{-} \rightarrow H(n=3)+H(n=1)
\end{aligned}
$$

In eq. (2), electrons in $\mathrm{H}^{-}$occupy the $1 \mathrm{~s}$ and affinity level, and latter electron transits to the vacant state with the quantum number of 3 in $\mathrm{H}^{+}$. Therefore, the energies and temperatures of $\mathrm{H}_{2}^{+}$and $\mathrm{H}^{+}$are detected in this experiment through the Balmer- $\alpha$ emission under the processes of eq. (1) and (2). The peak shift from 0 to $10 \mathrm{~V}$ of bias voltage is $20 \mathrm{pm}$ and the shift corresponds to $0.7 \mathrm{eV}$. On the other hand, the Doppler broadening of $30 \mathrm{pm}$ corresponds to more than $1 \mathrm{eV}$.

Detailed explanations of $\mathrm{H} \alpha \mathrm{LAS}$ are described elsewhere $^{12}$. In the case of H $\alpha$ LAS, temperature of 0.3 $\mathrm{eV}$ is obtained, though the first aim of this measurement is to compare the number density of thermalized $\mathrm{H}^{\circ}$ with Frank-Condon $\mathrm{H}^{\mathrm{o}}$ with the energy of $\sim 2.2 \mathrm{eV}$. The temperature is much lower than that obtained with HROES. One of the explanations is that $\mathrm{H}^{\mathrm{o}}(n=3)$ is detected in the case of HR-OES while $\mathrm{H}^{\mathrm{o}}(n=2)$ is the target of $\mathrm{H} \alpha$ LAS. Considering the Bolztmann distribution in the population of quantum state of $\mathrm{H}^{\mathrm{o}}(n)$, the $\mathrm{H}^{\mathrm{o}}(n=2)$ density predominates $\mathrm{H}^{\mathrm{O}}(n=3)$ density, and the temperature of $\mathrm{H}^{\mathrm{o}}(n=2)$ can be equilibrated with residual $\mathrm{H}^{\mathrm{o}}$ and $\mathrm{H}_{2}$ except $\mathrm{H}^{\mathrm{o}}(n=3)$ that may not be in thermal equilibrium.

\section{Flow of charged particles}

The dynamics of charged particles can be understood by investigating their flows. Flow analyses of electrons and $\mathrm{H}_{\mathrm{m}}{ }^{+}$ions, which are considered to be the parent particles of $\mathrm{H}^{-}$, have possibilities to reduce co-extracted electron current and enhance the $\mathrm{H}^{-}$extraction rate. For this reason, a directional Langmuir probe (DLP) with four probe tips is adopted to measure $\mathrm{H}^{-}$rich plasma ${ }^{15}$. The schematic structure is shown in Fig. 6. The DLP is movable in three-directions, and the entire body is rotatable around the axis of the probe stem. A schematic

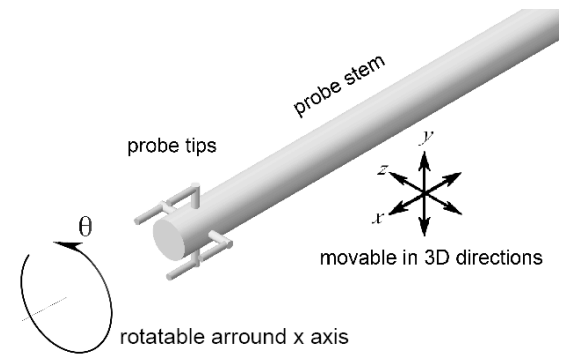

FIG. 6. Four-pin Langmuir probe and its movable directions

diagram of the DLP is shown in Fig. 6.

Figure 7(a) shows a typical polar distribution of negative saturation current obtained at $12 \mathrm{~mm}$ away from the PG without $\mathrm{Cs}$ seeding. Bias voltage of $2.4 \mathrm{~V}$ is applied and operational $\mathrm{H}_{2}$ pressure is $0.3 \mathrm{~Pa}$. The 0 degree angle corresponds to PG direction. Electrons and positive ions flows are obtained from the principal component of the FFT. Flow directions of positive ions at 
three different positions at 12, 19 and $26 \mathrm{~mm}$ away from the PG are indicated in Fig. 7(b). Flow directions of electrons are parallel to that of positive ions at each position. This shows ambipolar diffusion takes place.
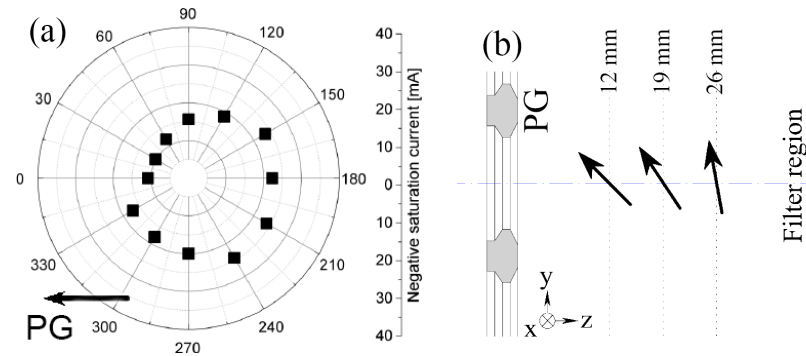

FIG. 7. (a) Polar distribution of negative saturation current in Cs seeded plasma and (b) flow direction of electron at $\mathrm{z}$ of 12,19 and $26 \mathrm{~mm}$.

Flow velocities of electron and positive ion are estimated from their saturation currents at the position 26 $\mathrm{mm}$ away from the PG. The electron and ion temperatures are estimated to be 0.3 and $0.65 \mathrm{eV}$, respectively. In order to compare which flux dominates the motion of charged particles, thermal velocities $\left(v_{\text {th }}\right)$, diffusion flux (mobility) and drift velocity $(\boldsymbol{E} \times \boldsymbol{B}$ drift) are obtained. The thermal velocities in $y$ and $z$ directions are $3.8 \times 10^{3}$ and $-2.4 \times 10^{2}$ $\mathrm{m} / \mathrm{sec}$, respectively. The $\boldsymbol{E} \times \boldsymbol{B}$ drift velocities in $y$ and $z$ directions are $4.2 \times 10^{3}$ and $-4.7 \times 10^{2} \mathrm{~m} / \mathrm{sec}$, respectively. The result shows the thermal and $\boldsymbol{E} \times \boldsymbol{B}$ drift velocities are of the same order in this case.

\section{E. $\mathrm{H}^{-}$flow and temperature}

Both of $\mathrm{H}^{-}$flow and temperature are measured by directional photodetachment (DPD) using DLP ${ }^{15}$. The temperature is also measured with detachment inducing CRD. It is well know that photodetachment is used to measure $\mathrm{H}^{-}$density. In the DPD, recovery time of $\mathrm{H}^{-}$after firing $\mathrm{Nd}-\mathrm{YAG}$ laser is applied to estimate the $\mathrm{H}^{-}$flow velocity and temperature. The $\mathrm{H}^{-}$ions in laser column release electrons and they are collected by a positively biased probe tip. Photodetachment signal, which constitutes the detached electrons, decays due to the charge recovery of $\mathrm{H}^{-}$surrounding the laser column. By irradiating the tips of photodetachment probe, the time evolution signal of negative saturation current is obtained.

Table 1 shows the $\mathrm{H}^{-}$temperatures and flow velocities in $\mathrm{y}$ and $\mathrm{z}$ directions ( $v_{\mathrm{y}}$ and $v_{\mathrm{z}}$, respectively) in Cs seeded plasma at the $\mathrm{H}_{2}$ pressure of $0.3 \mathrm{~Pa}$ and at the bias voltage of $2.5 \mathrm{~V}$. The flow directions are indicated in Fig. 9. It is noticeable that the $\mathrm{H}^{-}$flow direction is opposite to those of electrons and positive ions shown in Fig. 7. This feature

TABLE $1 . \mathrm{H}^{-}$temperature $\left(\mathrm{T}_{\mathrm{H}^{-}}\right)$and flow velocities in $\mathrm{y}\left(v_{\mathrm{y}}\right)$ and $\mathrm{z}$ directions $\left(v_{\mathrm{z}}\right)$.

\begin{tabular}{|c|c|c|c|}
\hline \hline & $\mathrm{z}=14[\mathrm{~mm}]$ & $\mathrm{z}=19[\mathrm{~mm}]$ & $\mathrm{z}=24[\mathrm{~mm}]$ \\
\hline Temperature & $0.20 \mathrm{eV}$ & $0.14 \mathrm{eV}$ & $0.19 \mathrm{eV}$ \\
\hline$v_{\mathrm{y}}$ & $-69 \mathrm{~m} / \mathrm{sec}$ & $-308 \mathrm{~m} / \mathrm{sec}$ & $-378 \mathrm{~m} / \mathrm{sec}$ \\
\hline$v_{\mathrm{z}}$ & $477 \mathrm{~m} / \mathrm{sec}$ & $152 \mathrm{~m} / \mathrm{sec}$ & $-137 \mathrm{~m} / \mathrm{sec}$ \\
\hline
\end{tabular}

may be attributed to the source regions of those particles. Especially, $\mathrm{H}^{-}$flow at $14 \mathrm{~mm}$ apart from $\mathrm{PG}$ directs from $\mathrm{PG}$ to driver region. The $\mathrm{H}^{-}$temperature is $0.12 \pm 0.03 \mathrm{eV}$ and the thermal velocity is one order larger then flow velocities.

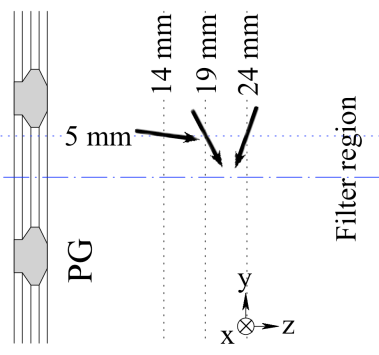

FIG. 9. $\mathrm{H}^{-}$flow directions at 14,19 and $24 \mathrm{~mm}$ apart from PG plane.

Another method to estimate is detachment-inducing CRD. In this method, the intensity of Nd-YAG is adjusted large enough to completely electron-detach $\mathrm{H}^{-}$along the laser path ${ }^{16}$. Penetration of $\mathrm{H}^{-}$from the surrounding plasma into this $\mathrm{H}^{-}$depleted channel increases the $\mathrm{H}^{-}$ density in the channel with time. Typical $\mathrm{H}^{-}$densities measured with normal CRD and those with detachment inducing CRD are shown in Fig. 10(a) and (b) respectively. Recovery time of $\mathrm{H}^{-}$density into the laser path is much
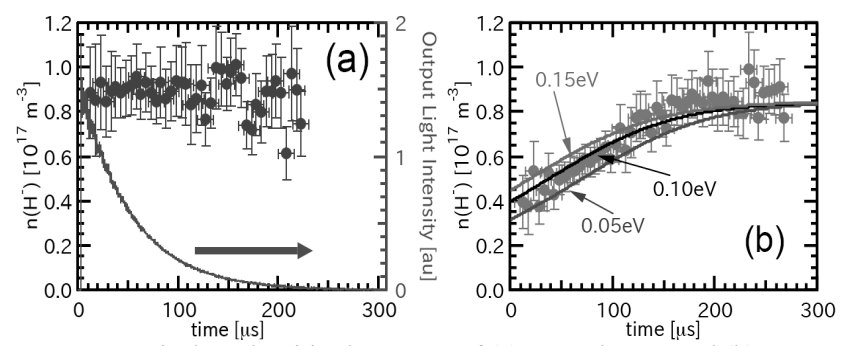

FIG. 10. Typical H- densities by means of (a) normal CRD and (b) detachment inducing CRD measurements.

slower than the reflection time in the cavity, and exponential rise time is observed in cavity signal. The $\mathrm{H}^{-}$ densities are obtained at $\mathrm{H}_{2}$ pressure of $0.3 \mathrm{~Pa}$, bias voltage of $2.4 \mathrm{~V}$ in $\mathrm{Cs}$ seeded plasma. The $\mathrm{H}^{-}$measured temperature is within the range of $0.5-1.5 \mathrm{eV}$ and is close to the result obtained with DPD.

\section{F. Beamlet experiment}

Two-dimensional distribution of $\mathrm{H}^{-}$density reduction due to beam extraction is provided by subtracting the CCD image of $\mathrm{H} \alpha$ during the beam extraction from the image before the beam extraction ${ }^{17}$. The subtracted image shows that $\mathrm{H}^{-}$extracted through the arrays of $\mathrm{PG}$ apertures shows the original $\mathrm{H}^{-}$density distribution spreading around the PG toward driver region as shown to be the bright part in Fig. 11. In this figure, density of extracted $\mathrm{H}^{-}$decreases by increasing the bias voltage, and it is consistent with the results obtained with CRD. To investigate how $\mathrm{H}^{-}$near the PG are extracted through extraction holes, a new device is installed near PG apertures at the period of an international collaboration between Italy and Germany. Figure 12 (a) and (b) show the device viewing from the top 


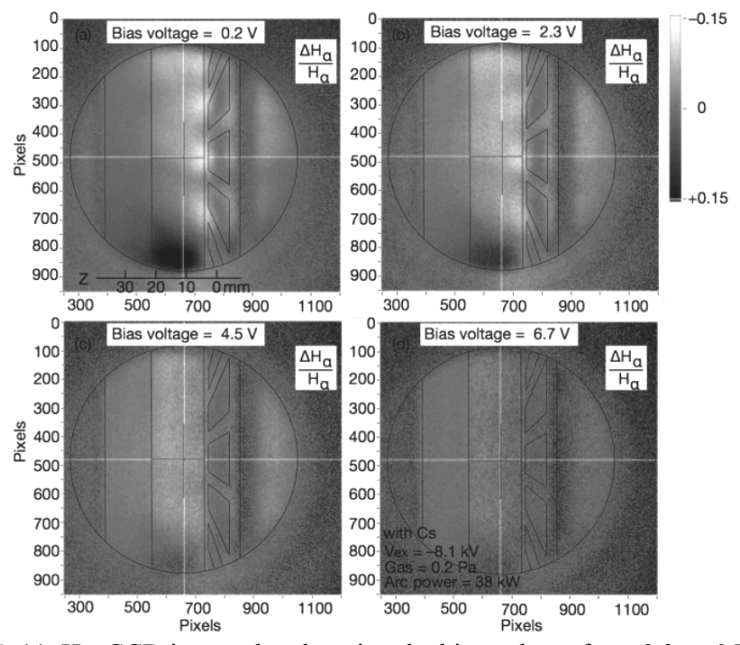

FIG. 11. H $\alpha$ CCD images by changing the bias voltage from 0.2 to $6.7 \mathrm{~V}$.

and front, respectively. Here the device is temporary called "beamlet eclipse shade". The shade is made of fine ceramics to avoid an electrical short circuit of plasma potential and is connected a movable stage set on the atmospheric side via a long ceramic stem. A window shaped mask is fixed on PG to fit the beam extraction area to a beamlet monitor called "mini-STRIKE" exported by NBI group of Istituto Gas Ionizzati del CNR, Consorzio RFX. Details of the mini-STRIKE are described elsewhere ${ }^{18}$.

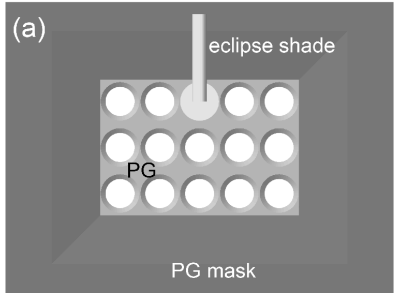

(b)

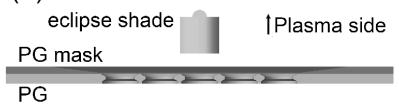

FIG. 12. Beamlet eclipse shade. (a) Top view and (b) front view.

Infrared images of beamlets on the mini-STRIKE with and without the eclipse shade are shown in Fig. 13(a) and (b), respectively. Notice that highest intensity part in those figures appears darker due to the analysis software. The shade put on an aperture at top center and the distance between the bottom of the shade cylinder and PG surface is $8.5 \mathrm{~mm}$. Comparing these images, a beamlet disappeared at shaded part and the beamlets surrounding the shade decrease the intensity. These are considered due to shading flux of parent particles of $\mathrm{H}^{-}$in extraction region. In addition, the peaks at the upper-most beamlet array slightly shift to upward and beamlets at the second and fourth column of the upper-most line shift on left-hand side. These displacements are possibly caused by beamlet interaction.

The eclipse shade method has large possibilities to investigate beamlet interaction and beamlet formation, as

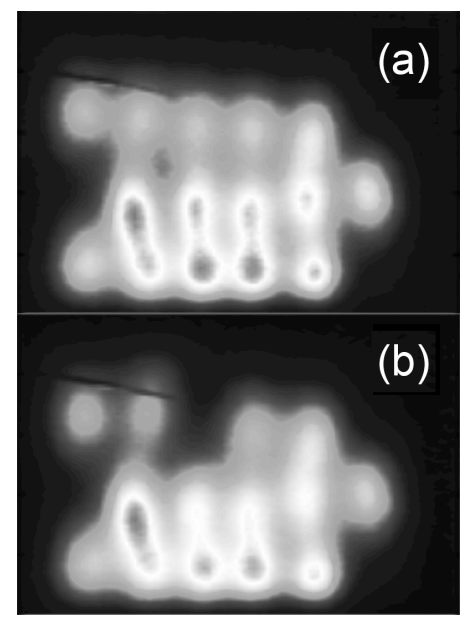

FIG. 13. IR images of $3 \times 5$ beamlets on mini-STRIKE (a) without and (b) with eclipse shade. Highest intensity at the peak part becomes darker due to intensity scale. A dark line running through two beamlet images at top-left is the shadow of a thermocouple.

well as its purpose of identifying the $\mathrm{H}$ - beam formation from plasma near the PG. Furthermore, it can be applicable to measure the production area of $\mathrm{H}^{-}$by replacing the thick ceramic cylinder to thin metal mesh or plate.

\section{DISCUSSION}

In the case of $\mathrm{Cs}$ seeded plasmas, $\mathrm{H}^{-}$ions are considered produced on the caesiated PG surface and emitted from sheath with the gap represented in Fig. 3(b). The $\mathrm{H}^{-}$ions transport in the $\mathrm{H}^{-}$-rich plasma with the energy of $0.5-1.5 \mathrm{eV}$, whose value is obtained with DPD and overneutralized CRD, and extracted after reaching the meniscus. To make the argument simple, let the plasma potential has the same value of bias voltage. In this case, incident energy of parent particle $\left(\varepsilon_{\text {in }}\right)$ and outgoing energy of $\mathrm{H}^{-}\left(\varepsilon_{\mathrm{H}^{-}}\right)$is kept following relation that $\varepsilon_{\mathrm{H}^{-}}=\varepsilon_{\text {in }}-e\left(\phi_{\mathrm{WF}}-\right.$ $\left.U_{\text {aff }}\right)^{20}$. Here $\phi_{\mathrm{WF}}$ and $U_{\text {aff }}$ are defined as work function and affinity energy of $\mathrm{H}^{-}$. When $\varepsilon_{\mathrm{H}^{-}}>0, \mathrm{H}^{-}$can escape from the surface. The relation can be expressed as $\varepsilon_{\text {in }}-\varepsilon_{\mathrm{H}^{-}}>$ $e\left(\phi_{\mathrm{WF}}-U_{\mathrm{aff}}\right)$, which means the kinetic-energy difference of incident particle and produced $\mathrm{H}^{-}$ion should be larger than the electron promotion energy from work function to affinity level at far enough from surface. Assuming the work function of caesiated PG, $\phi_{\mathrm{WF}}$, is $1.45 \mathrm{eV}$, the electron promotion energy becomes $0.70 \mathrm{eV}$ using the affinity level, $U_{\text {aff, }}$ of $0.75 \mathrm{eV}$. Our experimental result shows the $\mathrm{H}^{-}$energy is $0.5-1.5 \mathrm{eV}$, and this implies the incident energy of the parent particle $\left(\mathrm{H}^{\mathrm{o}}\right.$ or $\left.\mathrm{H}_{\mathrm{m}}{ }^{+}\right)$should be $1.25-2.25 \mathrm{eV}$ at least. Although the energy range is close to that of Frank-Condon hydrogen atom, H $\alpha$ LAS could not detected such energetic atoms. Closest incident energy of parent particle is observed only with HR-OES in this experiment, and the particle is considered $\mathrm{H}^{+}$of $\mathrm{H}_{2}{ }^{+}$ because of the response to the changing bias voltage. 
So far, it is not clear the reason why the other diagnostics cannot detect $\mathrm{H}_{\mathrm{m}}{ }^{+}$and/or $\mathrm{H}^{\circ}$. Especially, the flow obtained with DLP is equivalent to the energy much lower than $1.0 \mathrm{eV}$ by changing bias voltage. One of the large differences between HR-OES and the other diagnostic modules is the line-of-sights. The HR-OES views perpendicular direction to the arrays of electron deflection magnets (parallel to $y$ direction), and the others are parallel to the arrays (parallel to $x$ direction). If the $\mathrm{H}_{\mathrm{m}}{ }^{+}$is the dominant parent particle to $\mathrm{H}^{-}$, the drift direction is affected with strong magnetic field induced with electron deflection magnets, for instance $\boldsymbol{B} \times \nabla|\boldsymbol{B}|$ and $\boldsymbol{E} \times$ $\boldsymbol{B}$ drifts. Flow measurement using DLP is planed to carry out by changing the rotational axis to y direction.

As described in section III B, the fluxes of electron and positive ion concentrate to the cusp lines of EDM field. The electron flux compensates the extracted $\mathrm{H}^{-}$to conserve the charge neutrality with remained positive ions. Before and during extraction, the electrons come to the extraction region with positive ions via ambipolar diffusion and no significant flow has been observed. This indicates plasma has individual equilibriums before and during extraction and PG is exposed with constant flows of positive ions and electrons diffused from driver region. The ambipolar flow shown in Fig. 7(b) is partially blocked by beamlet eclipse shade set at $8.5 \mathrm{~mm}$ apart from PG. The beamlet intensity of $\mathrm{H}^{-}$is reduced down to $\sim 30 \%$ at the shade distance and it increases to $\sim 90 \%$ by separating the distance to $18.5 \mathrm{~mm}$. This eclipse method is simple and has a potential to obtain the transport mechanism of electrons and positive ions as well as $\mathrm{H}^{-}$by scanning the shade or changing the shape. The method is going to be applied to investigate beamlet interaction by changing the position of the shade.

\section{ACKNOWLEDGMENTS}

The authors would like to express their acknowledgements to the technical staff of LHD-NBI group for the excellent operations of NBI test stand. This work is supported by SOKENDAI Course-by-Course Education Program GGXX100, SOKENDAI Grant No. 70207003 GGRR003, JSPS KAKENHI Grant No. 25249134 and budget of NIFS No. ULRR702.

\section{REFERENCES}

${ }^{1}$ R.S. Hemsworth, J. H. Feist, M. Hanada et al., Rev. Sci. Instrum. 67, 1120 (1996).

${ }^{2}$ V. Antoni, P. Agostinetti, D. Aprile et al., Rev. Sci. Instrum. 85, 02B128 (2013).

${ }^{3}$ M. Hanada, N. Akino, Y. Endo et al., K. Usui, K. Watanabe and Larry R. Grisham, J. Plasma Fusion Res. 9, 208 (2010).

${ }^{4}$ A. Komori and T. Morisaki, J. Physics, Conf. Series 565, 012017 (2014).

${ }^{5}$ K. Tsumori, H. Nakano1, M. Kisaki et al., Rev. Sci. Instrum. 83, 02B116 (2012).

${ }^{6}$ K. Ikeda, H. Nakano, K. Tsumori et al., New Journal of Physics, 15, $103026(2013)$
${ }^{7}$ H. Nakano, K. Tsumori, M. Kisaki et al., AIP Conf. Proc.s, $\mathbf{1 5 1 5}$, 237, (2013).

${ }^{8}$ M. Kisaki, K. Tsumori, K. Ikeda et al., Rev. Sci. Instrum. 85, 02B131 (2014).

${ }^{9}$ S. Geng, K. Tsumori, H. Nakano et al., AIP Conf. Proc. 1655, 040014 (2015).

${ }^{10}$ S. Geng, K. Tsumori, H. Nakano et al., Plasma Fusion Res. 10, 3405016 (2015).

${ }^{11}$ M. Wada, T. Kenmotsu, M. Kisaki et al., 16th International Conference on Ion Sources (this conference), TuePE35, held at New York, USA (2015).

${ }^{12}$ H. Nakano, S. Nishiyama, M. Goto et al., AIP Conf. Proc., 1655, 020018, (2015).

${ }^{13}$ R. K. Janev, W. D. Langer, K. Evans, Jr., D. E. Post, Jr. "Elementary Processes in Hydrogen-Helium Plasmas Cross Sections and Reaction Rate Coefficients", Springer-Verlag Heidelberg, New York, London, Paris, Tokyo (Springer Series on Atoms and Plasmas Vol. 4).

${ }^{14}$ H. G. H. Dunn, B. van Zyl, and R. N. Zare, Phys. Rev. Let. 15, 610 (1965) and D. Auerbach, R. Cacak, R. Caudano, T. D. Gaily, C. J. Keyser, J. W. McGowan, J. B. A. Mitchell and S. F. J. Wilk, J. Physics B, Atom. Molec. Phys., 10, 3797 (1977).

${ }^{15}$ S. Geng, K. Tsumori, H. Nakano et al., 16th International Conference on Ion Sources (this conference), MonPS32, held at New York, USA (2015).

${ }^{16}$ H. Nakano, K. Tsumori, M. Shibuya et al., to be presented in 17th International Symposium on Laser-Aided Plasma Diagnostics, held in Hokkaido, Japan (2015).

${ }^{17}$ K. Ikeda, H. Nakano, K. Tsumori et al., Plasma and Fusion Res., 8, 1301036 (2013) and K. Ikeda, D. Wünderlich, U. Fantz, et al., 16th International Conference on Ion Sources (this conference), MonPE11, held at New York, USA (2015).

${ }^{18}$ P. Veltri, V. Antoni, P. Agostinetti et al., 16th International Conference on Ion Sources (this conference), MonPE11, held at New York, USA (2015) and G. Serianni F. Bonomo, M. Brombin et al., AIP Conf. Proc., 1515, 579 (2013).

${ }^{19} \mathrm{H}$. Nakano, K. Tsumori, M. Kisaki et al., to be presented in 68th Gaseous Electronics Conference / 9th International Conference on Reactive Plasmas, held in Honolulu, USA (2015). 20A. Pargellis and M. Seidl, Phys. Rev. 25, 4356 (1982). 